\title{
Hipertrofia mamaria virginal: caso clínico
}

\section{Virginal breast hypertrophy: case report}

Gabilondo Zubizarreta, F. J.*, Madariaga Romero, N.**, Meléndez Baltanas, J.**, Ayestarán Soto, J. ${ }^{* *}$, Caramés Estefanía, J.**

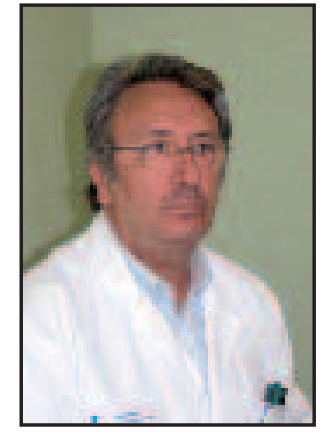

Gabilondo Zubizarreta, F.J.

\section{Resumen}

Presentamos un caso de hipertrofia virginal mamaria cuya aparición, controlada desde los 11 años, se estudió y diagnosticó mediante biopsia - aspiración como hipertrofia tipo filoides, siendo tratada quirúrgicamente mediante mamoplastia de reducción con patrón de Wise tradicional y colgajo horizontal bipediculado para el traslado del complejo areolar, que ha conservado la sensibilidad y la vascularización.

La intervención única se realizó a los 12 años de edad de la paciente y, tras 5 años de seguimiento postoperatorio, conserva la sensibilidad en las areolas y un aspecto de volumen y forma satisfactorio para la paciente, sin prótesis ni retoques de ningún tipo y con cicatrices aceptables.

\section{Palabras clave Hipertrofia virginal mamaria, mamo- plastia de reducción.}

Código numérico 521, 5249, 52102
We present a case of 12 years old patient, with a juvenile or virginal mammary hypertrophy and the postoperative result 5 years later without further recurrence and without any other treatment.

We decided to perform a breast reduction like Wise's style, with bipedicle horizontal flap to transport nipple-areola complex to the new location, and to keep sensibility.

Five years later, the outcome is satisfactory to the patient, with very pleasant breast volume and shape, discret scars, good sensibility and without recurrence nor other surgical treatment.

$\begin{array}{ll}\text { Key words } & \begin{array}{l}\text { Virginal breast hypertrophy, breast reduc- } \\ \text { tion. }\end{array} \\ \text { Numeral Code } & 521,5249,52102\end{array}$

\footnotetext{
* Jefe de Servicio.

** Médico Adjunto.

*** Médico Residente.

Servicio de Cirugía Plástica y Grandes Quemados del Hospital de Cruces, Servicio Vasco de Salud, Osakidetza. Baracaldo (Vizcaya). España.
} 


\section{Introducción}

El crecimiento mamario desproporcionado en niñas con niveles hormonales normales, en ausencia de gestación y sin finalidad funcional lactante, es conocido como hipertrofia virginal mamaria y al parecer, se debe a una respuesta exagerada del parénquima mamario a los estrógenos.

Todas las molestias físicas, dermatológicas y psíquicas que acompañan a este cuadro, a menudo asimétrico, suelen llevar a una situación límite a las pacientes a una edad muy temprana, que en el caso de la paciente que presentamos comenzó a los 11 años de edad.

Al parecer, conocida desde muy antiguo y citada en 1.669 en los escritos como "Philosophical Transcriptions", esta patología fue descrita y documentada posteriormente por Henry Albert en 1.910 en casos sin gestación, denominándosele como "Gigantomastia Juvenil o Hipertrofia Virginal Mamaria" (1).

Las propuestas terapéuticas hormonales han sido excluidas en estos casos por sus riesgos, optándose por las distintas alternativas quirúrgicas, desde la amputación mamaria, a la mastectomía subcutánea más colocación de prótesis $(2,3)$, o como en nuestro caso, mediante una gran reducción mamaria con traslado del complejo areolar, sin empleo de prótesis y utilizando el propio tejido mamario de la paciente como relleno.

\section{Caso clínico}

Paciente de 12 años de edad cuyo aspecto en el momento del diagnóstico es el que se muestra en la Figura 1; además de alteraciones dermatológicas, marcado plexo venoso subdérmico y asimetría de volumen, la paciente refería molestias físicas, psíquicas y dificultades para vestirse y para realizar ejercicios físicos, que hacían del cuadro una situación insostenible para el desarrollo de su vida cotidiana.

Se practicó examen anatomopatológico de biopsia obtenida por punción aspiración, encontrando una proliferación mamaria tisular tanto del estroma como del epitelio, con dificultad para diferenciarla de los patrones de fibroadenoma filoides Las propuestas terapéuticas hormonales se excluyeron, como ya hemos explicado, por sus posibles efectos secundarios. El tratamiento realizado consistió en una mamoplastia reductora siguiendo el patrón de Wise en el marcaje dérmico; tallamos un amplio colgajo horizontal bipediculado cuya desepidermización se aprovechó para disminuir el tamaño de las areolas, que trasladamos a su nueva ubicación conservando la sensibilidad y evitando así el injerto libre tipo Thoreck.

Empleamos el material autólogo de los colgajos a
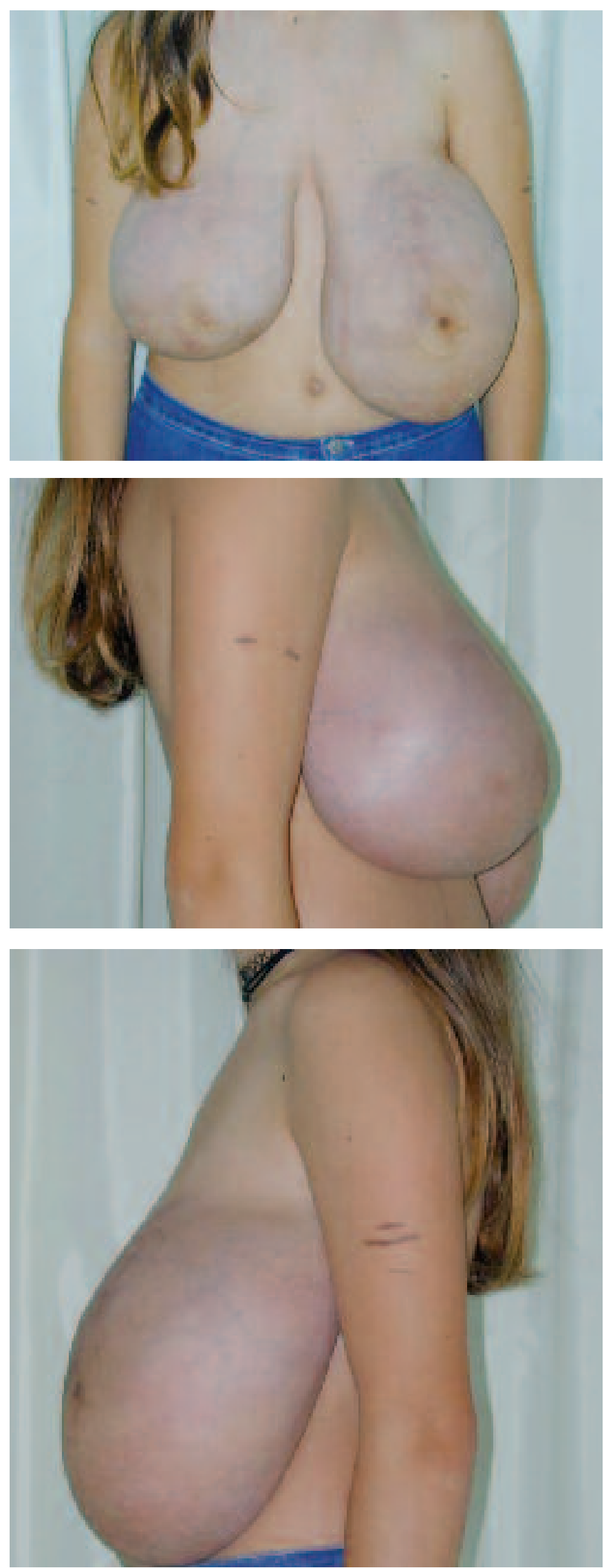

Fig. 1. Preoperatorio. Paciente de 11 años de edad. Véase en la imagen lateral izquierda la mama de mayor volumen.

modo de relleno, evitando de esta manera también el uso de prótesis. 


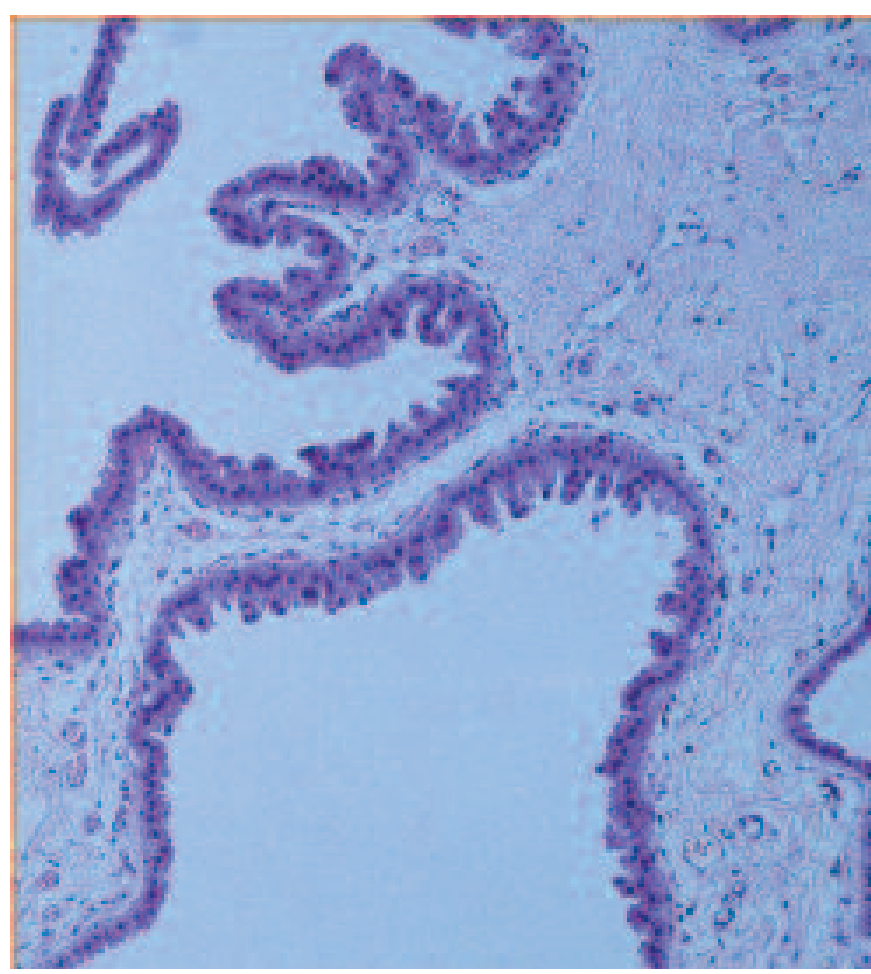

Fig. 2. Imagen del examen anatomopatológico de la biopsia por punción - aspiración (Hematosilina/Eosina, Microscopía óptica, $20 \mathrm{X}$ ): hipertrofia con patrón proliferativo del estroma y epitelio a modo de fibroadenoma filoides.

La resección en la mama derecha fue de $1.050 \mathrm{gr}$. de peso y en la izquierda llegó a los 1.800 gr.

Colocamos drenajes en ambas mamas que fueron retirados a las $48 \mathrm{~h}$. y la paciente fue dada de alta hospitalaria con una buena evolución, sin complicaciones y con el mismo diagnóstico anatomopatológico de hipertrofia con patrón proliferativo del estroma y epitelio a modo de fibroadenoma filoides (Fig.2).

El aspecto del resultado reciente, a los pocos meses de la intervención, podemos verlo en las Figuras 3: en él se aprecian unas mamas voluminosas aun para el tamaño y edad de la paciente, pero con una diferencia notable respecto a la situación previa.

El resultado más tardío (Fig.4), ya con 17 años y 6 meses de edad de la paciente, presenta un tamaño y una forma de las mamas más proporcionados a la edad y a las circunstancias de la paciente; ha mejorado el aspecto dérmico y vascular de sus mamas y no ha habido recurrencia del cuadro, mantiene la sensibilidad de los complejos areolares, las cicatrices son muy aceptables por su discreción y la satisfacción de la paciente le lleva a rechazar cualquier retoque de mejora.

\section{Discusión}

Ante la falta de una casuística suficiente, en una situación tan excepcional como la que viven las niñas con hipertrofia virginal mamaria verdadera (1), cuan-
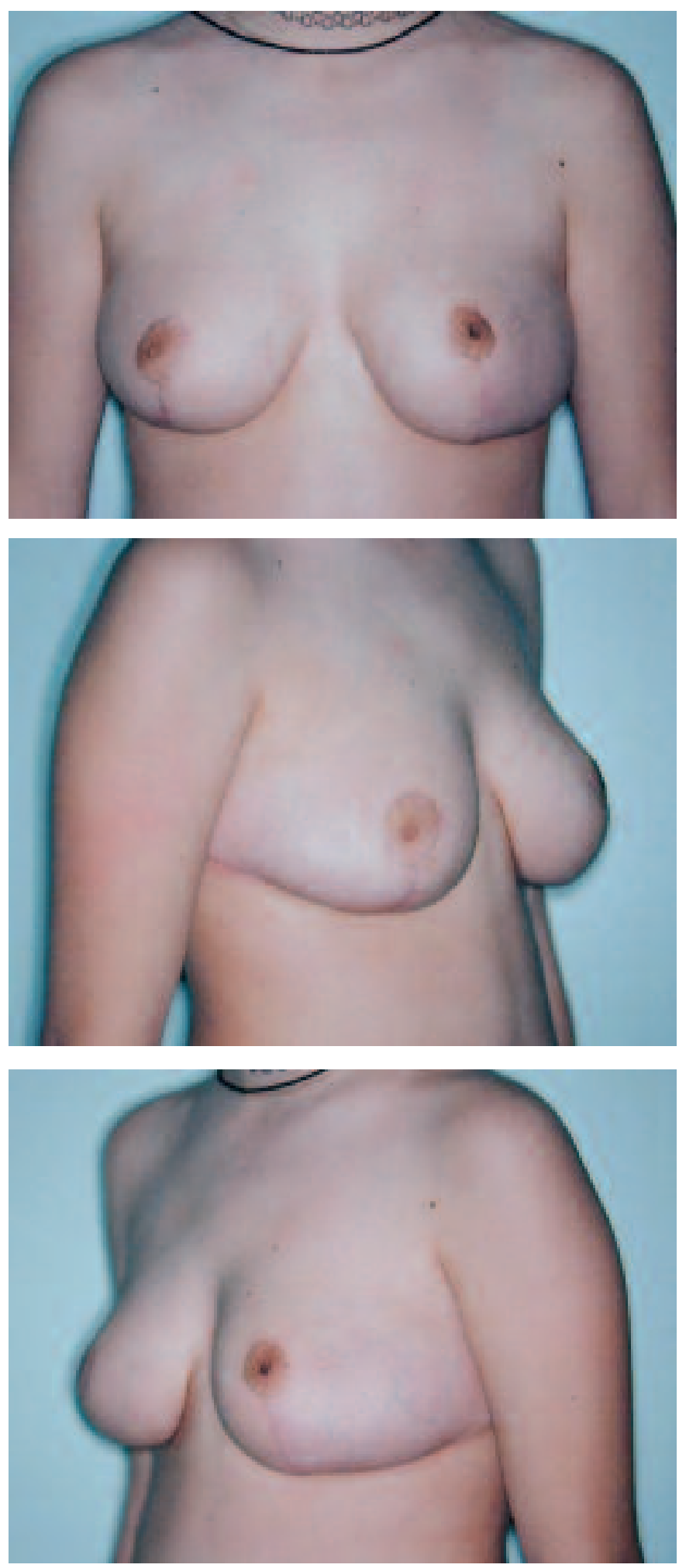

Fig. 3. Postoperatorio temprano.

do las exploraciones hormonales son normales y el cuadro requiere una solución pragmática, podemos valorar conservar la sensibilidad del complejo areolar practicando una reducción mamaria y evitando el uso de prótesis o la realización de amputaciones o mastectomías subcutáneas, ya que el futuro crecimiento de las pacientes nos permite obtener un volumen mamario que en la etapa adulta es razonable y pro- 

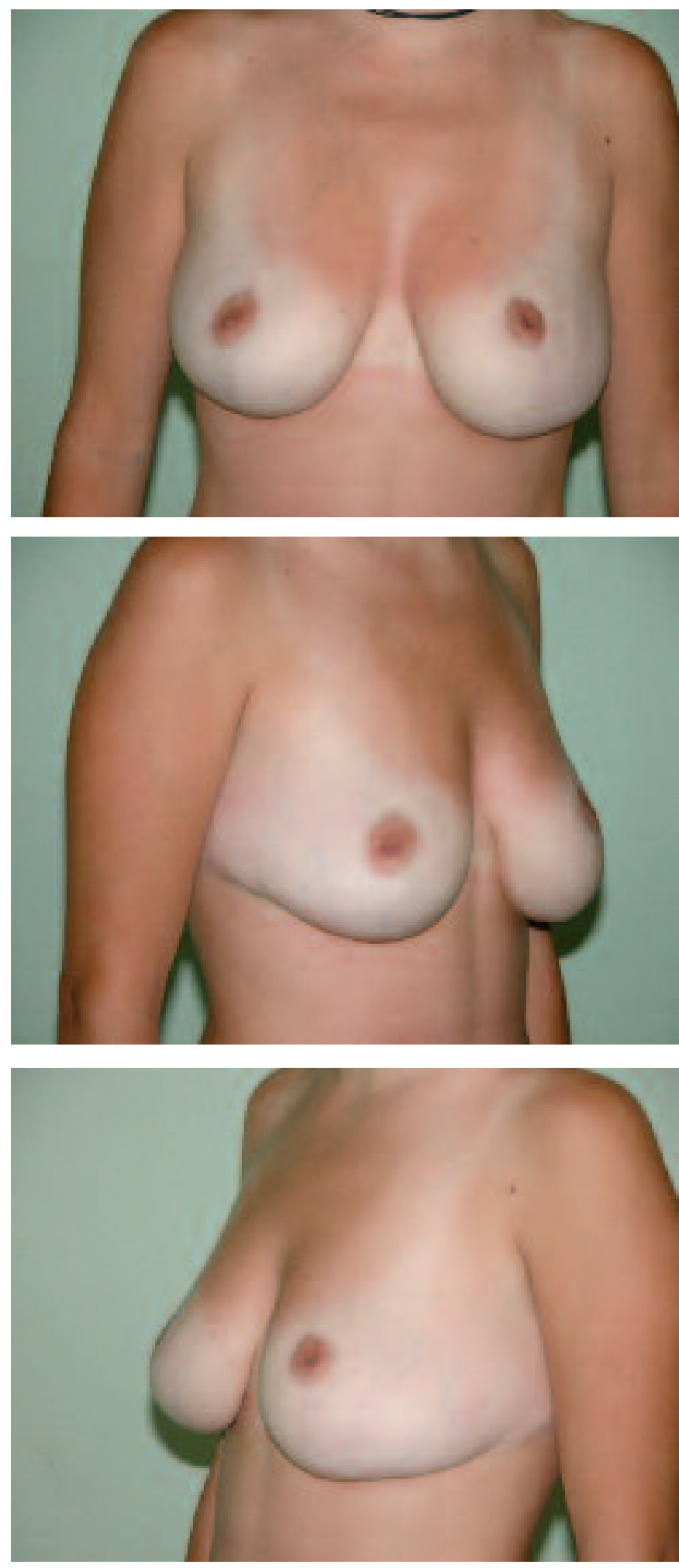

Fig. 4. Postoperatorio a los 5 años ( 17 años y 6 meses de edad de la paciente). Cicatrices discretas. porcionado, con lo que las pacientes mantienen sin recurrencia el resultado de la única y primera intervención.

Pensamos que de esta forma no es necesaria una amputación mamaria con o sin prótesis $(2,3)$, a la vez que obtenemos un buen resultado estético sin sacrificar la sensibilidad del complejo areolar.

Cada caso puede beneficiarse de técnicas diferentes, pero el fin funcional con mantenimiento de la sensibilidad, merece el esfuerzo.

\section{Conclusiones}

Las cicatrices en este tipo de cirugía son, con el tiempo, muy aceptables para las pacientes afectas de hipertrofia virginal mamaria y el resultado muy satisfactorio, evitando las complicaciones capsulares y los injertos libres de Thoreck.

Es importante aplicar la técnica quirúrgica en cada caso con la finalidad más funcional y preservando la sensibilidad del complejo areolar.

El resultado es satisfactorio tanto para la paciente como para el cirujano, cuando la intervención se realiza a una edad temprana, dado el impacto favorable para la paciente.

\section{Dirección del autor}

Dr. Francisco Javier Gabilondo Zubizarreta Servicio de Cirugía Plástica y Grandes Quemados Hospital de Cruces

Plaza de Cruces s/n. Baracaldo (Vizcaya). España e-mail:javier.gabilondo@osakidetza.net

\section{Bibliografía}

1. Albert, H.: "Diffuse idiopathic hypertrophy of the mammary glands in the female". J.A.M.A., 1910, 55: 1339.

2. Kupfer, D., Dingman, D., Broadbent, R.: "Juvenile Breast Hypertrophy: Report of a familiar Pattern and Review of the Literature". Plast. Reconstr. Surg., 1992, 90 (2): 303.

3. Cardoso de Castro, C., Aboudib, J.H., Salema, R.,Valladares, B.: "Massive Breast Hypertrophy in a young girl". Ann. Plast. Surg. 1990, 25: 497.

4. Greydanus, D. E., Parks, D. S., and Farrell, E.G.: "Breast disorders in children and adolescents". Pediatr. Clin. North Am., 1989, 36: 601 . 


\title{
Comentario al trabajo "Hipertrofia mamaria virginal: caso clínico"
}

\author{
Dra. Beatriz Berenguer Fröhner. Cirujano Plástico. Clínica La Luz. Madrid. España.
}

Uno de mis queridos profesores nos insistía con contagioso entusiasmo, que todo caso excepcional merece ser publicado, para que el siguiente que se encuentre con un caso similar tenga algo en lo que apoyarse.

En este sentido debemos agradecer a los autores el que nos relaten un caso de hipertrofia mamaria virginal verdaderamente excepcional en su presentación y resultado.

El Dr. Gabilondo ya nos ha deleitado en anteriores ocasiones con casos clínicos absolutamente singulares y me pregunto: ¿de dónde los saca? Probablemente se deba a que él es más disciplinado que otros cirujanos en comunicarlos.

Tal como se explica en el artículo, la hipertrofia mamaria virginal puede provocar importantes trastornos físicos y psíquicos a pacientes puberales, por lo que en estos casos, a diferencia de las reducciones mamarias habituales, es aconsejable adelantar la operación a edades más tempranas, y, valorando las particularidades del caso, elegir entre las técnicas disponibles. Por supuesto el objetivo es lograr el mejor resultado estético y funcional y, siempre que la situación lo permita, será preferible el tejido autólogo frente a la prótesis y las técnicas conservadoras de sensibilidad del complejo areola -pezón (incluyendo todos los diversos pedículos) frente al injerto libre. De esta forma, tal como concluyen los autores, en la mayoría de los casos se pueden obtener resultados muy satisfactorios para las pacientes y sus cirujanos. Recientemente traté a una paciente con una hipertrofia virginal mucho más leve, y al poco tiempo recibí el siguiente correo electrónico: "Doctora, muchas gracias por esta operación que me ha curado el físico y la cabeza...”. Seguro que la gratitud de la paciente del artículo era, proporcionalmente al tamaño del problema, mucho mayor.

Pero ya que en estos comentarios se nos exige una lectura crítica (constructiva), quisiera hacer algunas anotaciones:

Los autores comienzan con una bonita introducción histórica a este raro problema, pero son un poco parcos en la descripción de tan interesante caso. Empezando por la anamnesis: ¿El crecimiento desproporcionado se presentó de forma brusca o gradual tras la telarquia? ¿Cómo fue de rápido, semanas, meses? ¿Hubo algún proceso intercurrente? Se han descrito casos primaros y otros casos secundarios p. ej. a trasplantes renales, tumores o tirotoxicosis (1). ¿Cuándo aparecieron los trastornos dermatológicos asociados y cuáles eran en concreto? La literatura recoge diversas alteraciones dermatológicas asociadas a la hipertrofia virginal que incluyen hipersensibilidad, eritema intenso, edema e hiperplasia verrucosa. Algunos de estos síntomas pueden mejorar con tratamiento con Tamoxifeno durante el seguimiento, a la espera de la reducción mamaria (2) ¿Se investigaron antecedentes familiares para descartar la forma familiar de hipertrofia virginal?

En cuanto a la exploración física, además del aspecto es muy importante detallar la palpación. ¿Cuál era la consistencia: blanda, firme, pétrea? ¿La hipertrofia era difusa o existían masas localizadas? ¿Presentaba adenopatías axilares?

Toda esta información es muy importante a la hora de establecer el diagnóstico diferencial entre las diferentes causas de macromastia puberal benigna: fibroadenoma juvenil (54\%), mastopatía fibroquística (22\%), hipertrofia virginal (13\%) y cistoadenoma filoides $(7 \%)$, y descartar los rarísimos tumores mamarios malignos puberales que pueden confundirse con la hipertrofia virginal, como el linfoma mamario o el cistosarcoma filoides. (Todas estas patologías pueden ser unilaterales o bilaterales).

Para el estudio de confirmación la prueba de imagen de elección en este grupo de edad es la ecografía que puede resultar sumamente útil; se desaconseja la mamografía por la dosis de radiación y porque es difícil de interpretar con tejidos jóvenes tan densos. En caso de que persistan dudas, siempre se debe comprobar el diagnóstico preoperatorio mediante biopsia (aspirativa, como en este caso, o incisional).

La mayoría de las macromastias puberales benignas parecen relacionarse con una hipersensibilidad a las hormonas gonadales, por lo que tanto el aspecto macroscópico como el microscópico pueden ser parecidos, como en el caso que se presenta, que en el resumen en castellano se describe como "hipertrofia virginal de tipo filoides". No obstante, este detalle puede resultar confuso, si no se explican las características de este otro raro tumor, y quizá sea mejor omitirlo del todo, tal como se ha hecho en el resumen en inglés.

Por último, creo que los autores han sido muy habilidosos y la paciente muy afortunada, pero de la experiencia de un caso no se puede generalizar que "...las pacientes mantienen sin recidiva (que no recurrencia), el resultado de la única y primera intervención.” En mi experiencia compartida, y la de otros autores, algunas veces recidivan, incluso años después (3). Un colega me comentó en una reunión que tuvo que reducir a la misma paciente $; 3$ veces! El sobrecrecimiento de la hipertrofia virginal puede prolongarse durante años, por lo tanto, si se opta por una técnica conservadora de tejido mamario antes de estabilizado el crecimiento, debe advertirse a la paciente y a sus padres de la posibilidad de que recidive la macromastia (además de todas las posibles complicaciones de cualquier reducción mamaria). A pesar de ello creo que está muy justificada la intervención precoz, y felicito de nuevo a los autores por su resultado a corto y largo plazo.

Bibliografía

1. Griffith JR. "Virginal breast hypertrophy”. J Adolesc Health Care. 1989 Sep;10(5):423.

2. O'Hare PM, Frieden IJ. "Virginal breast hypertrophy". Pediatr Dermatol. 2000 Jul-Aug;17(4):277.

3. Pulzl P, Schoeller T, Tzankov A, Wechselberger G. "Unilateral breast enlargement 5 years after reduction mammaplasty". Aesthetic Plast Surg. 2005 Sep-Oct;29(5):404. 


\section{Respuesta al comentario de la Dra. Beatriz Berenguer Fröhner}

\section{Dr. Francisco Javier Gabilondo Zubizarreta}

Sorprendido con algunos contenidos de la Dra. Beatriz Berenguer Fröhner, confieso que aunque no era nuestro objetivo, deleitar a los lectores con nuestras aportaciones, nos alegra ese efecto añadido.

En su extenso comentario a nuestra parca exposición, nos da ocasión a responder a sus inquietudes al tratarse de un caso en que, por ser médicos los padres de la paciente, la información es de calidad probada a pesar de la rareza del mismo.

Remitida desde la Unidad de Endocrinología Pediátrica, nos informaron de que el crecimiento de ambas mamas se desarrolló en meses $(10-12)$ de forma progresiva y la consulta a nuestro Servicio, al año del comienzo del proceso, se hizo aproximadamente a los 12 años de la paciente, no acompañándose de otra patología o proceso, ni tratamiento alguno medicamentoso. Tampoco hay antecedentes ni se ha presentado en su entorno familiar posterior ningún caso similar.

En la exploración, además del aspecto, que es muy demostrativo en las imágenes, no se encontraron ni se palparon adenopatías, siendo llamativo lo desdibujado de los complejos areolares, la intensidad del trazado venoso y algunas estrías de distensión, junto a lesiones eritemato-eczematosas en la piel del contacto mamario con el tronco, siendo la palpación mamaria de una distribución difusa, consistente e indolora.
No se realizaron mamografías convencionales a la paciente, sí un estudio de imagen con resonancia magnética nuclear, y tanto la biopsia por aspiración como el estudio de las piezas de resección ante la ausencia de signos de malignidad y los patrones mixtos proliferativos llevaron a un diagnóstico que excluye, las tumoraciones malignas similares y otros procesos que en la literatura se han confundido con la hipertofia juvenil o virginal verdadera y que cita la Dra. Berenguer oportunamente.

Por último, reiteramos que hasta la actualidad nuestra paciente no presenta recurrencia o recidiva del proceso, coincidiendo que un caso no sirve para la generalización, aunque hay publicaciones confusas en las que se etiquetan de hipertrofia juvenil o virginal otras patologías que no lo son, sin más pretensión pronóstica por tratarse de un caso clínico tan sólo.

Amablemente le recomiendo a mi colega que en lo concerniente a la recidiva versus recurrencia, consulte el diccionario de María Moliner en su página 888 y si no es fuente suficientemente solvente o autorizada, en la página. 1746 de la XXI edición del de la Real Academia Española de la Lengua, sobre el uso médico de ambos términos.

Esperando que mi comentario a los suyos haya sido de su satisfacción, le envío un cordial saludo. 